Article

\title{
Production Sustainability via Supermarket Location Optimization in Assembly Lines
}

\author{
Masood Fathi ${ }^{1, *(1)}$, Amir Nourmohammadi ${ }^{1}\left(\mathbb{D}\right.$, Morteza Ghobakhloo $^{2}$ and Milad Yousefi ${ }^{3}$ \\ 1 Department of Production and Automation Engineering, University of Skövde, P.O. Box 408, \\ SE-541 28 Skövde, Sweden; amir.nourmohammadi@his.se \\ 2 Department of Industrial Engineering, Minab Higher Education Center, University of Hormozgan, \\ Bandar Abbas P.O. Box 7981634314, Iran; ghobakhloo.morteza@gmail.com \\ 3 Department of Industrial Engineering and Transportation, Universidade Federal do Rio Grande do \\ Sul-UFRGS, Porto Alegre 90035-190, Brazil; yousefi.milad@gmail.com \\ * Correspondence: maood.fathi@his.se; Tel.: +46-(0)-500-448575
}

Received: 12 May 2020; Accepted: 7 June 2020; Published: 9 June 2020

\begin{abstract}
Manufacturers worldwide are nowadays in pursuit of sustainability. In the Industry 4.0 era, it is a common practice to implement decentralized logistics areas, known as supermarkets, to achieve production sustainability via Just-in-Time material delivery at assembly lines. In this environment, manufacturers are commonly struggling with the Supermarket Location Problem (SLP), striving to efficiently decide on the number and location of supermarkets to minimize the logistics cost. To address this prevalent issue, this paper proposed a Simulated Annealing (SA) algorithm for minimizing the supermarket cost, via optimally locating supermarkets in assembly lines. The efficiency of the SA algorithm was tested by solving a set of test problems. In doing so, a holistic performance index, namely the total cost of supermarkets, was developed that included both shipment cost and the installation cost across the assembly line. The effect of workload balancing on the supermarket cost was also investigated in this study. For this purpose, the SLP was solved both before and after balancing the workload. The results of the comparison revealed that workload balancing could significantly reduce the total supermarket cost and contribute to the overall production and economic sustainability. It was also observed that the optimization of material shipment cost across the assembly line is the most influencing factor in reducing the total supermarket cost.
\end{abstract}

Keywords: assembly line; optimization; production sustainability; supermarket location; simulated annealing

\section{Introduction}

Manufacturers nowadays are realizing the significant economic benefits of sustainable production practices [1,2]. Production sustainability denotes the application of economically-efficient manufacturing and management processes that reduce manufacturing costs and wastes while pursuing operational efficiency [3,4]. In their quest for achieving production sustainability, modern manufacturers need to materialize the concept of the sustainable production line $[5,6]$. Nowadays, assembly lines are widely used in different industries due to their advantages in effectively dealing with high volume production [7]. The efficiency of an assembly line, to a great extent, depends on its configuration and design. Thus, many managers are eager to find a suitable solution method to deal with the long-term decision problem of assembly line design from the sustainable workload distribution and material supply point of view [8].

The task of workload distribution at assembly lines is known as the assembly line balancing problem (ALBP) [9]. The ALBP concerns the proper allocation of work tasks to a set of stations so that 
all the stations have an equal amount of work while considering and satisfying all the operational and technological requirements [10].

Material supply is concerned with efficient delivery of material to the point of use to avoid any excessive cost as well as to avoid shortages at stations [11]. The most known philosophy for efficient material delivery is called Just-in-Time (JIT), where material should only be delivered where needed, when needed, and with the necessary amount. Recently, a new concept known as Just-in-Sequence (JIS) delivery is also introduced and widely used in the industry. Following JIS, the supplier sorts the parts into bins in a specific order facilitating the work of the production operators so that parts are withdrawn by operators according to the production sequence. Both JIT and JIS concepts contribute to production sustainability by reducing delivery cycles through a detailed plan for logistics operations [12].

With the introduction of JIT and JIS concepts, a large number of manufacturers around the world have applied the supermarket concept in feeding materials to assembly lines. In a logistics definition, supermarkets are decentralized storage areas close to the assembly lines acting as intermediate storage for stations. Generally, human-driven or automated tow trains are used for material delivery from supermarkets to stations through milk-run delivery. Tow trains operate based on a predetermined schedule and feed parts from supermarkets to the stations that are located on their predetermined routes across an assembly line. The use of supermarkets and tow trains allow parts to be frequently delivered in small lots, which leads to the reduction of inventory level at the stations and removal of long-distance deliveries from the centralized storage [13,14]. These features provided by the use of supermarkets significantly contribute to transportation sustainability in the manufacturing environment [15]. However, and because of the general space scarcity across the shop floor, an increase in the number of supermarkets would be associated with an overall higher logistics cost. Alternatively, lowering the number of supermarkets below the minimum requirement increases the travel distance, leading to an increase in transportation costs across the assembly line [12,16]. In such circumstances, the Supermarket Location Problem (SLP), which concerns the problem of identifying the suitable location and number of supermarkets, is commonly regarded as a long-term decision problem within the supermarket-based sustainable material delivery context [17].

This study aims to contribute to the production sustainability background by investigating the effect of assembly line balancing on overall logistics costs, the issue that has received lesser attention to date. Since both the SLP and ALBP are complex combinatorial optimization problems $[18,19]$, there has been a growing trend toward using metaheuristics to address them. Considering that the SLP is less developed compared to ALBP, this study contributes to the literature by emphasizing SLP and benefiting from the established results on ALBP widely available within the literature to perform the necessary comparisons and benchmarking. In this regard, this study proposes a Simulated Annealing (SA) algorithm to address the SLP during the strategic decision-making phase to promote production sustainability via logistics cost efficiency. In particular, the study benefits from two distinct cost-related indices related to the use of supermarket for material delivery at assembly lines, namely supermarket installation cost and material transportation cost. The efficiency of the proposed SA algorithm was tested against the optimum solution obtained by mathematical optimization. The present study not only investigates and explains how contemporary manufacturers can achieve a higher level of production efficiency via optimally positioning supermarkets across assembly lines but also mathematically compares the role and importance of supermarket installation cost and material transportation cost in the achievement of logistics cost efficiency and sustainability.

The remainder of the paper is organized as follows. Section 2 provides a literature review on SLP. In Section 3, the description of the SLP as a long-term decision problem as well as its assumptions and mathematical formulation are presented. Section 4 describes the proposed solution approach to deal with the SLP. The computational results are presented in Section 5, while the analysis of results and complementary discussions are outlined in Section 6. Section 7 offers the concluding remarks and discusses the limitations of the study. 


\section{Literature Review}

Review of the literature shows that although ALBP has been studied for decades, and a rich literature exists on this topic, there are less than a handful of studies addressing the SLP while pursuing production sustainability. It is worth mentioning that there are also some similarities between the SLP addressed in this study and the studies related to the location selection problem and inventory management. However, this review is limited to only the most related studies where the SLP was addressed. The researchers interested may refer to Chen et al. [20] and Zeng et al. [21] for further reading about the two related topics mentioned above. Moreover, the studies by Li et al. [22] and Eghtesadifard et al. [23] provide a comprehensive review of recent trends and advances on ALBP. To provide an insight into the SLP literature, a review of the most relevant studies is provided below.

Battini et al. [24] proposed a step-by-step decision support procedure to address the SLP while taking the transportation and inventory costs of supermarkets into consideration. Based on the procedure proposed for different part components, the best feeding strategy ranging from the complete centralized, supermarket, or complete decentralized areas was found. In this study, it is assumed that each supermarket feeds several assembly lines, while in practice, many real-world manufacturers employ more than one supermarket to feed each assembly line. Emde and Boysen [16] applied the Dynamic Programming (DP) approach to cope with the SLP while minimizing the supermarket installation and the tow train transportation costs. The results of the study showed that the proposed DP could optimally solve the industrial-size SLP within polynomial computational time. Through comprehensive simulation experiments, the quality of the solutions has been validated from operational and economic aspects while compared to the centralized warehouse area. The proposed DP in this study is applicable when supermarkets could be placed anywhere on the factory layout, which might not be feasible due to space limitations on the shop floor. Alnahhal and Noche [18] addressed the SLP by applying a Genetic Algorithm (GA) to optimize the total transportation and installation costs while tow trains were used for milk-run material delivery. The performance of the proposed GA was tested by solving a set of problems and comparison against the optimal solutions found by a mathematical optimization model. Despite the results obtained being motivating, no discussions or insights in terms of transportation and installation costs were provided in this study.

Nourmohammadi and Eskandari [25] developed a hierarchical approach based on mathematical models to optimize the assembly line design in terms of workload balancing and SLP. The models proposed were validated by solving a set of standard test problems using GAMS/CPLEX solver. The results obtained showed the efficiency of the models in solving problems up to a certain size. Despite the complexity of the SLP, no meta-heuristic algorithm was proposed in this study to address large-size problems. Nourmohammadi et al. [26] investigated the effect of the stations' demand variations on the SLP solutions. To this attempt, the authors developed a mathematical model for the stochastic SLP by assuming the station demands to follow a normal distribution where supermarkets had to keep safety stocks to respond to the demand variations. The results of the study showed that the mathematical optimization approach proposed could optimally solve SLP while minimizing the shipment, inventory, and installation costs. Considering the complexity of SLP, the optimization approach proposed only tested on problems of limited-size, and no huristic/met-heuristic approach was developed to tackle large size problems. Nourmohammadi et al. [27] addressed the ALBP and SLP using a hierarchical stochastic mathematical model while assuming that the task times and demands follow the normal distribution. The proposed model was validated by solving a set of test problems and computational analysis. Despite the satisfying performance of the model on solving small size problems, due to the stochastic and complex nature of the SLP, there is no guarantee that large-size problems could efficiently be solved using the same model. Thus, the lack of an efficient approximation method/algorithm is felt in this study.

Zhou and Tan [28] addressed the SLP by considering the limited capacity and the utilization rate of supermarkets. The authors proposed an algorithm based on Differential Evolution (DE) to optimize the installation/operation cost of the supermarket as well as the transportation cost. The results 
of the study showed that the proposed DE is capable of finding good solutions for the considered problems. The performance of the DE was tested through comparison with an existing GA approach. However, no comparison with exact methods (i.e., mathematical model) was performed to evaluate the quality of the solutions obtained and making a reliable judgment about the efficiency of the DE developed. Recently, Nourmohammadi et al. [17] addressed the joint supermarket location and transport vehicle selection problem by assuming different transportation modes for parts feeding. The authors proposed a mixed-integer programming model and a Hybrid Genetic Algorithm (HGA) with a variable neighborhood search to address large-sized problems with the objectives of minimizing the procurement and shipment costs of vehicles as well as the installation cost of supermarkets. To test the efficiency of the proposed HGA, a set of standard test problems was solved by the algorithm, the mathematical model, and another heuristic algorithm. The computational results showed that the HGA could provide quality solutions in a reasonable time. This study was merely concentrated on SLP and did not investigate the effect of the ALBP on the solutions.

The current study proposes an efficient SA algorithm customized for solving the SLP problem. In contrast with the previous studies, it is assumed that several supermarkets can be used to feed a single assembly line, and only specific places in the lineside can be chosen for establishing supermarkets. The effect of the workload balancing was also investigated on the supermarket cost, which was considered to be the sum of both the installation cost and the transportation cost. To this aim, SLP was solved twice (i.e., before and after addressing the ALBP) and the supermarket costs are compared for a reliable conclusion on the effect of line balancing on the supermarket cost.

\section{Problem Description}

A general description of the SLP, its assumptions and mathematical formulation are presented in this section.

\subsection{Description of SLP}

Assuming a straight assembly line where supermarkets supply stations $w=1, \ldots, W(W=$ number of stations) with $d_{w}$ bins of parts, stations across the assembly line are designated by $\left(x_{w}, y_{w}\right)$. Alternatively, the locations for supermarkets are demonstrated by $\left(X_{s}, Y_{s}\right) ; s=1, \ldots, P S P$ where PSP denotes the possible number of places for establishing supermarkets. To determine the distance traveled by tow trains for supplying bins of parts to various stations, the three variables of (1) distance between supermarkets to stations, (2) distance from the station to station, and (3) the distance from the station back to the supermarket are calculated. In other words, dist $t_{s v w}$ that indicates the collective distance train travels from supermarket $s$ to station $v$, then from station $v$ to $w$ and back from station $w$ to supermarket $s$ is calculated via the following equation.

$$
\operatorname{dist}_{s v w}=\left\{\left|X_{s}-x_{v}\right|+\left|Y_{s}-y_{v}\right|\right\}+\left\{\left|x_{v}-x_{w}\right|+\left|y_{v}-y_{w}\right|\right\}+\left\{\left|X_{s}-x_{w}\right|+\left|Y_{s}-y_{w}\right|\right\}
$$

Considering the above description, the SLP strives to discover the optimal number of supermarkets from a possible number of locations. More importantly, SLP attempts to identify the stations that should be supplied via each supermarket, considering certain assumptions. Figure 1 shows a layout of an assembly line where two supermarkets are established for material delivery to stations by tow trains.

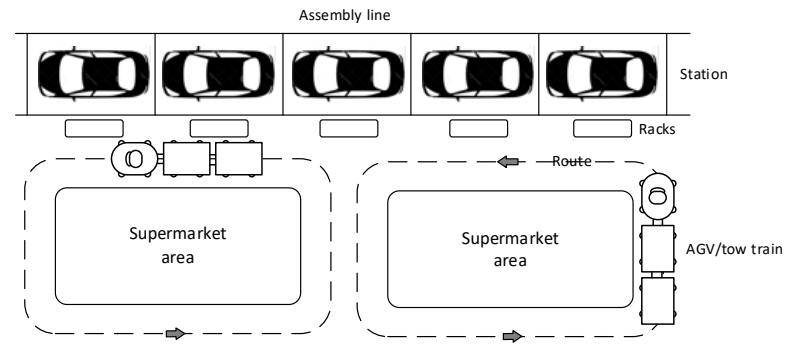

Figure 1. Material delivery to assembly line via the supermarket. 


\subsection{The SLP Assumptions}

According to the literature (e.g., [16,28]), the main assumptions of SLP can be summarized as follows.

- The order (sequence) through which each supermarket visits stations is consecutive. This means, for example, a particular supermarket is not allowed to serve stations 1, 2, and 5 if stations 3 and 4 are served by other supermarkets.

- The optimal number and location of supermarkets should be selected from the candidate supermarket places. Supermarket capacity, when it comes to serving the stations, is assumed to be limited. Thus, additional supermarket positions have to open when the capacity limitation of a particular supermarket is reached.

- $\quad$ The bins holding parts are identical and standardized in size.

- The arrangement of stations follows a straight-line pattern. Therefore, and considering the shop floor space limitation, supermarkets are assumed to be located as close to the stations as possible. Consistently, it is assumed that the supermarket locations smoothly scatter next to the shooter racks of workstations.

\subsection{Problem Formulation}

The SLP problem considered in this study is similar to the one addressed in Alnahhal and Noche [18] in terms of assumptions and constraints. To better understand the problem, the mathematical programming model of the considered SLP in this study is presented below. The notations used in the model are given in Table 1.

Table 1. List of notations.

\begin{tabular}{cl}
\hline Notations & \\
\hline Indices: & station index \\
$w, v$ & supermarket index \\
Parameters & number of stations \\
$W$ & possible supermarket places \\
$P S P$ & supermarket installation cost \\
$S I C$ & demand for station $k$ per shift \\
$d e m_{w}$ & $x$ and $y$ coordinates of station $w$ \\
$x_{w}, y_{w}$ & $x$ and $y$ coordinates of supermarket $s$ \\
$X_{S}, Y_{s}$ & total demands of all stations from $v$ to $w$ \\
$T d e m_{v w}$ & distance AGV/tow train travels from supermarket $s$ to feed all stations from $v$ to $w ;$ \\
$d i s t_{s v w}$ & dist $t_{s v w}=\left\{\left|X_{S}-x_{v}\right|+\left|Y_{s}-y_{v}\right|\right\}+\left\{\left|x_{v}-x_{w}\right|+\left|y_{v}-y_{w}\right|\right\}+\left\{\left|X_{S}-x_{w}\right|+\left|Y_{s}-y_{w}\right|\right\}$ \\
$S U C$ & the shipment unit cost that occurs by moving one bin for one unit of distance \\
$D S D_{v w}$ & demand standard deviation for all stations from $v$ to $w$ \\
$C A P$ & supermarket capacity in terms of the number of bins \\
$S L$ & service level for the probability of not exceeding the supermarket capacity \\
Decision variables & \\
$Z_{s v w} \in(0,1)$ & 1 if supermarket $s$ feeds all stations from $v$ to $w ;$ otherwise 0 \\
$N S \in$ Integer & number of supermarkets installed \\
\hline
\end{tabular}

According to the above notations, the Mixed Integer Programming (MIP) formulation of SLP is given as follows.

$$
\begin{gathered}
\text { Minimize TC= } \sum_{s=1}^{P S P} \sum_{v=1}^{W} \sum_{w=v}^{W} S U C \times T^{\prime} d e m_{v w} \times d i s t_{s v w} \times Z_{s v w}+S I C \times N S \\
\sum_{S=1}^{P S P} \sum_{v=1}^{W} \sum_{w=v}^{W} Z_{S v w}=N S
\end{gathered}
$$




$$
\begin{gathered}
\sum_{s=1}^{P S P} \sum_{v=1}^{u} Z_{s v u}=\sum_{s=1}^{P S P} \sum_{w=u+1}^{W} Z_{s(u+1) w} ; \forall u=1, \ldots, W-1 \\
\sum_{v=1}^{W} \sum_{w=v}^{W} Z_{s v w} \leq 1 ; s=1, \ldots, P S P \\
\left(T_{d e m} m_{v w}+S L \times D S D_{v w}\right) \times Z_{s v w} \leq C A P ; \forall s=1, \ldots, P S P, \forall v=1, \ldots, W, \forall w=v, \ldots, W \\
N S \geq 1
\end{gathered}
$$

In this model, the objective function aims to minimize the total cost (TC) of supermarkets, which includes the shipment cost (SC) and the installation cost (IC) as calculated by the first and the second terms in Equation (2), respectively. Equation (3) determines the number of supermarkets established for parts supply to stations. Equation (4) ensures that the established supermarkets feed each group of stations known as cells. Equation (5) guarantees that each group of stations/cells is supplied by only one supermarket. The capacity limitation of the established supermarkets is satisfied by Equation (6). This inequality ensures that the average demands of a group of stations assigned to each supermarket, considering a certain safety level and demand fluctuation at stations, does not exceed the supermarket capacity. Finally, Equation (7) ensures that at least one supermarket is established for material supply to stations.

\section{The Proposed Solution Approach}

In this study, a SA algorithm is proposed to address the SLP. To better clarify the solution procedure, the general structure for solving the SLP is proposed in Figure 2.

\subsection{Simulated Annealing for SLP}

SA is a generic probabilistic metaheuristic algorithm that was first introduced by Kirkpatrick et al. [29]. This algorithm is capable of producing near-optimum solutions with the polynomial cost for NP-hard problems. In SA, the search process is similar to the local search method in the sense that it starts with an initial solution in the search space and iteratively investigates the neighbors of each state and accepts a better solution until it has achieved a near-optimal solution. However, SA can skip local traps by accepting worse solutions with a certain probability. As suggested in the literature (e.g., [30-32]), the acceptance probability in this study is calculated according to the Equation presented below.

$$
P(\Delta E, T)=\exp \left(\frac{\Delta E=f\left(I S^{\prime}\right)-f(I S)}{T}\right)
$$

where $T$ is the annealing temperature that imitates the reducing temperature, which is applied in the metal annealing process, and $f(I S)$ and $f\left(I S^{\prime}\right)$ are the fitness function values of the current and the candidate neighbor solutions, respectively. This probability function is reduced as $T$ is updated by a cooling schedule. To accept the worse solution, a number between 0 and 1 is randomly generated. The worse solution is accepted if and only if the generated number is smaller than or equal to $P$; otherwise, it is rejected.

\subsubsection{Initial Temperature and Cooling Rate}

The SA algorithm starts with an initial randomly generated vector of integer numbers. The initial temperature is crucial, given its sheer impact on the identification of good solutions. The selection of the starting temperature is based on the nature of the problem and the related literature. Based on some pilot studies, we have set the value of the starting temperature equal to 1000 to keep the intensification property of the SA algorithm as precise and gentle as possible. In addition, the cooling schedule is calculated using the Equation presented below.

$$
T_{\gamma+1}=T_{\gamma}-1
$$


where $T_{\gamma}$ is the temperature in the iteration $\gamma$.

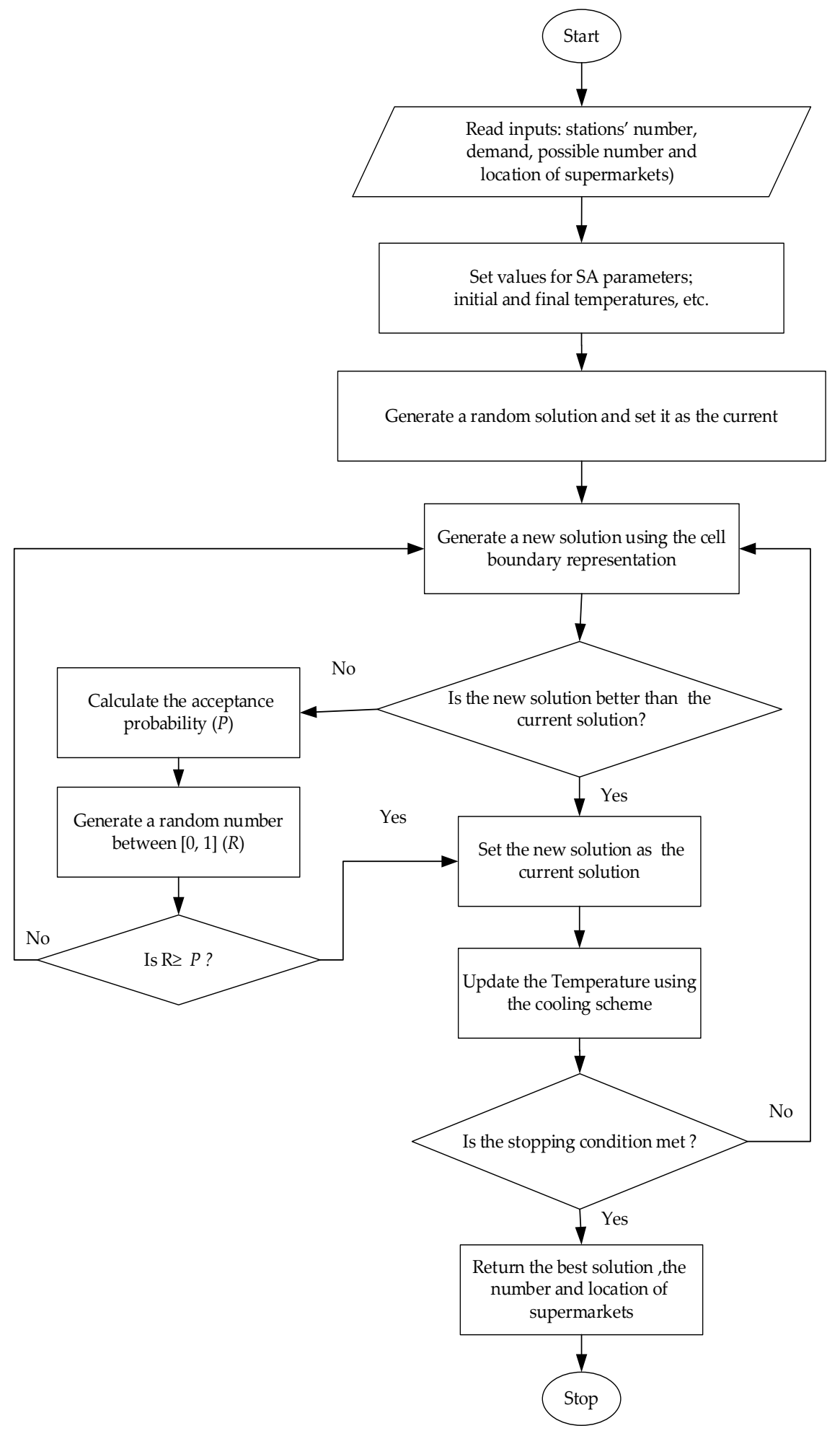

Figure 2. The applied solution procedure to solve for the Supermarket Location Problem (SLP).

\subsubsection{Solution Representation}

Two representations types, namely "Prüfer number representation" and "cell boundary", exist in the SLP literature, see Alnahhal and Noche [18]. In this study, the second type of representation is used to enhance the algorithm's search efficiency. This representation, which is also called "rightmost 
station", constitutes a string of integer numbers representing a series of stations to be served by each supermarket. The number in each cell represents the last station to which each supermarket will serve. Figure 3 shows the cell boundary representation for a ten-supermarket instance. According to this figure, for instance, supermarket 4 will serve stations 6 to 9 where 6 and 9 are the leftmost and the rightmost stations/boundaries of supermarket 4 , respectively.

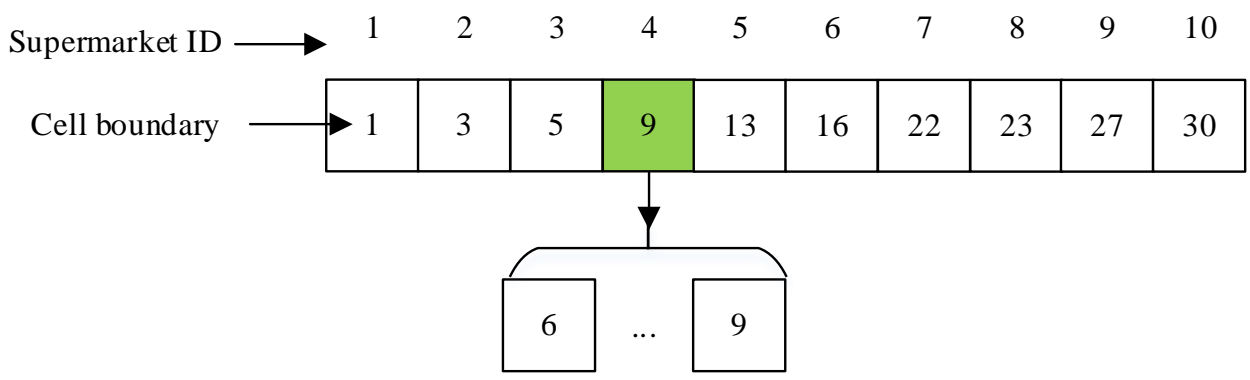

Station range served by supermarket 4

Figure 3. The cell boundary representation.

\subsubsection{The Neighborhood Search Operator}

To search the neighborhood of each solution, the mutation operator is applied. To this aim, a randomly selected cell boundary is chosen. Based on the position of this selected cell, the cell boundary is randomly changed without exceeding its right and left most boundaries (see cell boundary representation depicted in Figure 3). In this study, after some experiments and pilot tests, the mutation rate is set to 0.3 . The pseudocode of the mutation operator is provided in Table 2.

Table 2. The pseudocode of the mutation operator.

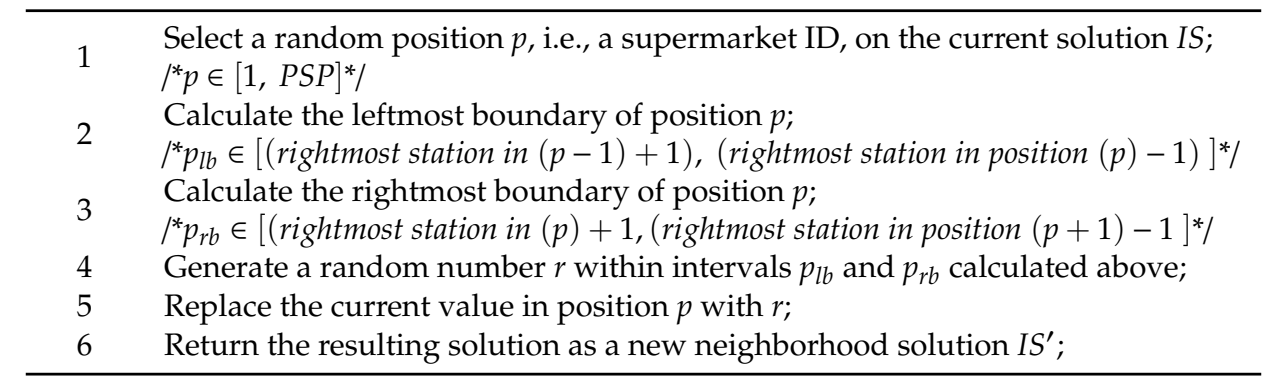

Figure 4 provides a visual overview of the mutation operator used for the neighborhood search. In this figure, for instance, the 4 th cell boundary can be altered to be any value from 6 to 8 and from 10 to 12 as the left and rightmost boundaries, respectively.

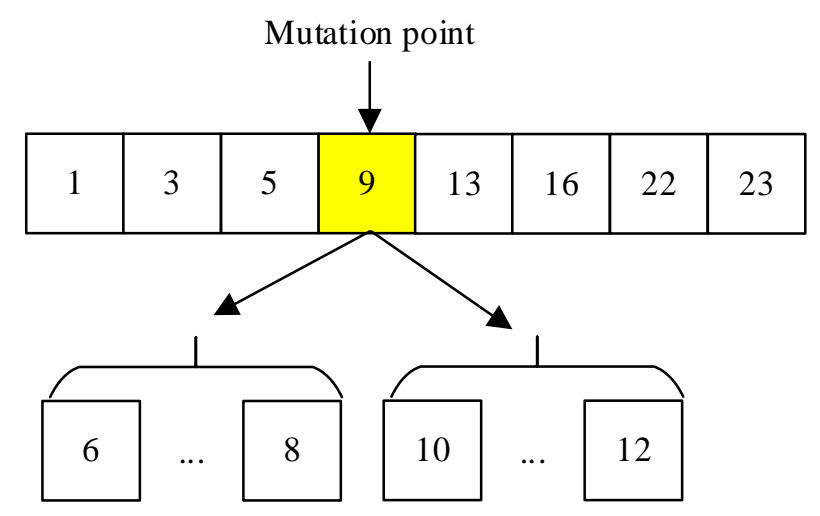

Figure 4. The mutation operator for neighborhood search. 


\subsubsection{The Evaluation Function and Termination Condition}

In addressing the SLP, we aim to minimize the total logistics cost, including (1) the cost of the weighted distance traveled by tow trains and (2) the installation cost of supermarkets. The weighted distance cost is calculated through multiplying the total distance traveled from a particular supermarket for supplying a station group (cell), by the entire demand of this cell, i.e., $Q_{s v w}=T_{d e m_{v w}} \times d i s t_{s v w}$. Thus, the objective function of the SLP known as total cost (TC) of the supermarket, which is composed of the supermarkets' transportation and installation costs, is calculated using the equation presented below.

$$
\operatorname{Min} T C=\sum_{s=1}^{P S P} \sum_{v=1}^{W} \sum_{w=v}^{W} S U C \times Q_{s v w}+S I C \times N S
$$

where USC, SIC and NS are the unit shipment cost, the supermarket installation cost, and the number of supermarkets, respectively.

The SA algorithm continues until a stopping condition is met. In this study, the algorithm is terminated when the maximum number of iterations is reached.

\subsubsection{Pseudocode of the Proposed Algorithm}

To better understand the proposed SA algorithm, the pseudocode of the algorithm is provided in Table 3.

Table 3. The pseudocode of the proposed Simulated Annealing (SA) algorithm for SLP.

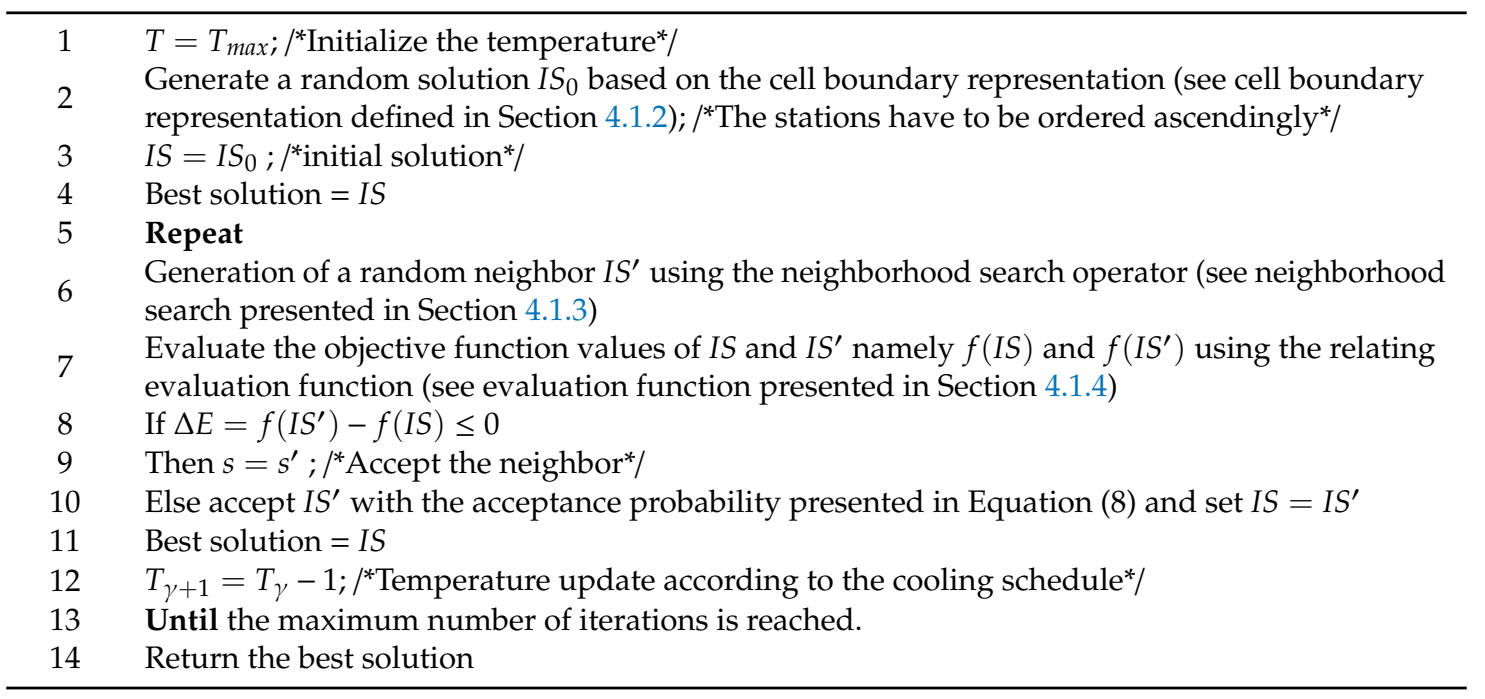

\section{Computational Results}

To assess the efficiency of the proposed SA algorithm, several test problems with differentiae characteristics are solved. It is worth mentioning that the test problems are taken from the "Assembly Line Balancing Data Sets \& Research Topics" (see the data sets here, https://assemblyline-balancing.de/) and adapted to this study by adding the information about the material demand. The problems are divided into small, medium, and large size based on the number of assembly tasks. All the problems are solved for two scenarios in terms of possible supermarket locations in the assembly lines (i.e., $P S P=4$ and 5). To further analyze and assess the effect of the installation cost on the solution, two different values are considered for the supermarket installation cost (i.e., SIC $=500$ and 1000). It is also assumed that the demand for tasks is known in advance in terms of the number of bins. The demand for each task in bins is generated using uniform distribution $U(1,10)$. Assuming that the capacity of supermarkets is limited, the maximum capacity of each supermarket was chosen as equal to 150 bins. The shipment unit cost (SUC) is considered to be 10 units of cost. 
To make a reliable comparison, considering the defined assumptions, all the problems were solved by both CPLEX and the SA algorithm proposed. No time limit was given for CPLEX and therefore, all the problems were solved to optimality. The stopping condition of the SA was set as when the final temperature of zero is reached using the relating cooling schedule. The SA algorithm was coded in Matlab R2018a and run on a computer with $2.4 \mathrm{GHz}$ Core i7 CPU.

Table 4 represents the comparison of the best solutions obtained by the SA with the optimal solutions obtained by CPLEX in terms of the total cost (TC). The gap between the obtained solution by $\mathrm{SA}$ and the optimum solution is also reported for each problem solved. All the problems were solved for two possible supermarket places (i.e., $P S P=4$ and 5) and two different supermarket installation costs (i.e., $S I C=500$ and 1000 unit cost). To view the stochastic treatment of the algorithm, the results are obtained after running the SA on the considered test problems 10 times, and the best-found solutions are reported.

Table 4. The results obtained by the CPLEX and the proposed SA for the test problems.

\begin{tabular}{|c|c|c|c|c|c|c|c|c|c|c|c|c|c|}
\hline \multirow{2}{*}{ Problem } & \multirow{2}{*}{$C T$} & \multicolumn{6}{|c|}{$S I C=500$} & \multicolumn{6}{|c|}{$S I C=1000$} \\
\hline & & SA & CPLEX & Gap (\%) & SA & CPLEX & Gap (\%) & SA & CPLEX & Gap (\%) & SA & CPLEX & Gap (\%) \\
\hline Jackson & 9 & 6928 & 6928 & 0.0 & 6900 & 6900 & 0.0 & 8300 & 8300 & 0.0 & 8300 & 8300 & 0.0 \\
\hline \multirow[t]{2}{*}{ Mitchell } & 14 & 14,480 & 14,480 & 0.0 & 14,480 & 14,480 & 0.0 & 16,480 & 16,480 & 0.0 & 16,698 & 16,480 & -1.3 \\
\hline & 15 & 14,480 & 14,480 & 0.0 & 14,440 & 14,440 & 0.0 & 16,480 & 16,480 & 0.0 & 16,940 & 16,480 & -2.7 \\
\hline \multirow[t]{2}{*}{ Sawyer } & 41 & 20,840 & 20,840 & 0.0 & 20,584 & 20,584 & 0.0 & 22,840 & 22,840 & 0.0 & 23,084 & 22,840 & -1.1 \\
\hline & 48 & 20,435 & 20,435 & 0.0 & 20,440 & 20,408 & -0.2 & 22,435 & 22,435 & 0.0 & 22,940 & 22,408 & -2.3 \\
\hline \multirow[t]{2}{*}{ Gunther } & 44 & 25,240 & 25,240 & 0.0 & 24,190 & 24,190 & 0.0 & 27,240 & 27,240 & 0.0 & 26,690 & 26,690 & 0.0 \\
\hline & 49 & 24,550 & 24,550 & 0.0 & 23,000 & 23,000 & 0.0 & 26,550 & 26,550 & 0.0 & 25,500 & 25,500 & 0.0 \\
\hline \multirow[t]{2}{*}{ Kilbridge } & 62 & 33,320 & 33,320 & 0.0 & 32,152 & 32,152 & 0.0 & 35,320 & 35,320 & 0.0 & 34,652 & 34,652 & 0.0 \\
\hline & 69 & 32,000 & 32,000 & 0.0 & 31,276 & 31,276 & 0.0 & 34,000 & 34,000 & 0.0 & 33,776 & 33,776 & 0.0 \\
\hline
\end{tabular}

To investigate the effect and influence of the workload balancing on the solution of SLP, all the test problems are solved before and after balancing the workload (i.e., considering the feasible and the optimum solution for ALBP). Tables 5 and 6 show the obtained results by SA for two possible supermarket places (i.e., $P S P=4$ and 5 ) on the considered test problems by considering the two different supermarket installation costs (i.e., SIC $=500$ and 1000 unit cost). The solutions are reported and compared in terms of $I C=\sum_{S=1}^{P S P} \sum_{v=1}^{W} \sum_{w=v}^{W} S U C \times Q_{s v w}$ and $S C=S I C \times N S$ as well as the $T C$ representing the installation cost of the supermarkets (IC), the shipment cost (SC), and the total cost (TC), respectively. Each problem is solved two times, once with a feasible solution and once with the optimum solution for the ALBP. 
Table 5. The computational results of applying feasible and optimal assembly line balancing problem (ALBP) + SLP on different test problems for SIC $=500$.

\begin{tabular}{|c|c|c|c|c|c|c|c|c|c|c|c|c|c|c|c|c|c|c|}
\hline \multirow{3}{*}{ No. } & \multirow{3}{*}{ Problem } & \multirow{3}{*}{$C T$} & \multicolumn{8}{|c|}{$P S P=4$} & \multicolumn{8}{|c|}{$P S P=5$} \\
\hline & & & \multicolumn{4}{|c|}{ Feasible ALBP + SLP } & \multicolumn{4}{|c|}{ Optimal ALBP + SLP } & \multicolumn{4}{|c|}{ Feasible ALBP + SLP } & \multicolumn{4}{|c|}{ Optimal ALBP + SLP } \\
\hline & & & $m$ & IC & $S C$ & $T C$ & $m$ & IC & $S C$ & $T C$ & $m$ & IC & $S C$ & $T C$ & $m$ & IC & $S C$ & $T C$ \\
\hline 1 & \multirow[b]{2}{*}{ Jackson } & 7 & 9 & 2000 & 5600 & 7600 & 8 & 2000 & 5400 & 7400 & 9 & 2000 & 5680 & 7680 & 8 & 2000 & 5400 & 7400 \\
\hline 2 & & 9 & 7 & 1500 & 5700 & 7200 & 6 & 1500 & 5428 & 6928 & 7 & 2000 & 5424 & 7424 & 6 & 2000 & 4900 & 6900 \\
\hline 3 & \multirow{2}{*}{ Mitchell } & 14 & 10 & 2000 & 13,500 & 15,500 & 8 & 2000 & 12,480 & 14,480 & 10 & 2000 & 13,520 & 15,520 & 8 & 2500 & 11,980 & 14,480 \\
\hline 4 & & 15 & 9 & 2000 & 12,700 & 14,700 & 8 & 2000 & 12,480 & 14,480 & 9 & 2500 & 11,960 & 14,460 & 8 & 2500 & 11,940 & 14,440 \\
\hline 5 & \multirow{2}{*}{ Buxey } & 36 & 11 & 2000 & 24,140 & 26,140 & 10 & 2000 & 23,455 & 25,455 & 11 & 2500 & 22,140 & 24,640 & 10 & 2500 & 21,360 & 23,860 \\
\hline 6 & & 41 & 9 & 2000 & 22,040 & 24,040 & 8 & 2000 & 21,360 & 23,360 & 9 & 2500 & 21,084 & 23,584 & 8 & 2500 & 20,626 & 23,126 \\
\hline 7 & \multirow{2}{*}{ Sawyer } & 41 & 9 & 2000 & 19,640 & 21,640 & 8 & 2000 & 18,840 & 20,840 & 9 & 2500 & 18,480 & 20,980 & 8 & 2500 & 18,084 & 20,584 \\
\hline 8 & & 48 & 8 & 2000 & 18,840 & 20,840 & 7 & 2000 & 18,435 & 20,435 & 8 & 2500 & 18,138 & 20,638 & 7 & 2500 & 17,940 & 20,440 \\
\hline 9 & \multirow{2}{*}{ Gunther } & 44 & 15 & 2000 & 25,720 & 27,720 & 12 & 2000 & 23,240 & 25,240 & 15 & 2500 & 23,920 & 26,420 & 12 & 2500 & 21,690 & 24,190 \\
\hline 10 & & 49 & 11 & 2000 & 22,660 & 24,660 & 11 & 2000 & 22,550 & 24,550 & 11 & 2500 & 20,820 & 23,320 & 11 & 2500 & 20,500 & 23,000 \\
\hline 11 & \multirow{2}{*}{ Kilbridge } & 62 & 10 & 2000 & 32,823 & 34,823 & 9 & 2000 & 31,320 & 33,320 & 10 & 2500 & 30,000 & 32,500 & 9 & 2500 & 29,652 & 32,152 \\
\hline 12 & & 69 & 9 & 2000 & 31,380 & 33,380 & 8 & 2000 & 30,000 & 32,000 & 9 & 2500 & 29,080 & 31,580 & 8 & 2500 & 28,776 & 31,276 \\
\hline 13 & \multirow{2}{*}{ Arcus1 } & 3786 & 22 & 2000 & 81,100 & 83,100 & 21 & 2000 & 78,620 & 80,620 & 22 & 2500 & 75,340 & 77,840 & 21 & 2500 & 69,860 & 72,360 \\
\hline 14 & & 4454 & 21 & 2000 & 79,080 & 81,080 & 18 & 2000 & 72,300 & 74,300 & 21 & 2500 & 71,840 & 74,340 & 18 & 2500 & 64,900 & 67,400 \\
\hline 15 & \multirow{2}{*}{ Tonge } & 160 & 26 & 2000 & 87,750 & 89,750 & 23 & 2000 & 79,680 & 81,680 & 26 & 2500 & 75,300 & 77,800 & 23 & 2500 & 70,380 & 72,880 \\
\hline 16 & & 168 & 24 & 2000 & 82,440 & 84,440 & 22 & 2000 & 78,440 & 80,440 & 24 & 2500 & 75,150 & 77,650 & 22 & 2500 & 68,860 & 71,360 \\
\hline
\end{tabular}

Table 6. The computational results of applying feasible and optimal ALBP + SLP on different test problems for SIC = 1000.

\begin{tabular}{|c|c|c|c|c|c|c|c|c|c|c|c|c|c|c|c|c|c|c|}
\hline \multirow{3}{*}{ No. } & \multirow{3}{*}{ Problem } & \multirow{3}{*}{$C T$} & \multicolumn{8}{|c|}{$P S P=4$} & \multicolumn{8}{|c|}{$P S P=5$} \\
\hline & & & \multicolumn{4}{|c|}{ Feasible ALBP + SLP } & \multicolumn{4}{|c|}{ Optimal ALBP + SLP } & \multicolumn{4}{|c|}{ Feasible ALBP + SLP } & \multicolumn{4}{|c|}{ Optimal ALBP + SLP } \\
\hline & & & $m$ & IC & $S C$ & $T C$ & $m$ & IC & $S C$ & $T C$ & $m$ & IC & $S C$ & $T C$ & $m$ & IC & $S C$ & $T C$ \\
\hline 1 & \multirow{2}{*}{ Jackson } & 7 & 9 & 3000 & 6200 & 9200 & 8 & 3000 & 5960 & 8960 & 9 & 3000 & 6440 & 9440 & 8 & 3000 & 5960 & 8960 \\
\hline 2 & & 9 & 7 & 3000 & 5700 & 8700 & 6 & 2000 & 6300 & 8300 & 7 & 2000 & 6872 & 8872 & 6 & 2000 & 6300 & 8300 \\
\hline 3 & \multirow[b]{2}{*}{ Mitchell } & 14 & 10 & 4000 & 13,500 & 17,500 & 8 & 4000 & 12,480 & 16,480 & 10 & 5000 & 12,456 & 17,456 & 8 & 5000 & 11,698 & 16,698 \\
\hline 4 & & 15 & 9 & 4000 & 12,700 & 16,700 & 8 & 4000 & 12,480 & 16,480 & 9 & 5000 & 12,000 & 17,000 & 8 & 5000 & 11,940 & 16,940 \\
\hline 5 & \multirow[b]{2}{*}{ Buxey } & 36 & 11 & 4000 & 24,340 & 28,340 & 10 & 4000 & 23,455 & 27,455 & 11 & 5000 & 22,360 & 27,360 & 10 & 5000 & 21,360 & 26,360 \\
\hline 6 & & 41 & 9 & 4000 & 22,040 & 26,040 & 8 & 4000 & 21,360 & 25,360 & 9 & 5000 & 21,060 & 26,060 & 8 & 5000 & 20,626 & 25,626 \\
\hline 7 & \multirow{2}{*}{ Sawyer } & 41 & 9 & 4000 & 19,640 & 23,640 & 8 & 4000 & 18,840 & 22,840 & 9 & 5000 & 18,480 & 23,480 & 8 & 5000 & 18,084 & 23,084 \\
\hline 8 & & 48 & 8 & 4000 & 18,840 & 22,840 & 7 & 4000 & 18,435 & 22,435 & 8 & 5000 & 18,138 & 23,138 & 7 & 5000 & 17,940 & 22,940 \\
\hline 9 & \multirow{2}{*}{ Gunther } & 44 & 15 & 4000 & 25,580 & 29,580 & 12 & 4000 & 23,240 & 27,240 & 15 & 5000 & 23,604 & 28,604 & 12 & 5000 & 21,690 & 26,690 \\
\hline 10 & & 49 & 11 & 4000 & 22,660 & 26,660 & 11 & 4000 & 22,550 & 26,550 & 11 & 5000 & 21,020 & 26,020 & 11 & 5000 & 20,500 & 25,500 \\
\hline 11 & \multirow{2}{*}{ Kilbridge } & 62 & 10 & 4000 & 32,822 & 36,822 & 9 & 4000 & 31,320 & 35,320 & 10 & 5000 & 30,000 & 35,000 & 9 & 5000 & 29,652 & 34,652 \\
\hline 12 & & 69 & 9 & 4000 & 31,380 & 35,380 & 8 & 4000 & 30,000 & 34,000 & 9 & 5000 & 29,080 & 34,080 & 8 & 5000 & 28,776 & 33,776 \\
\hline 13 & \multirow{2}{*}{ Arcus1 } & 3786 & 22 & 4000 & 81,520 & 85,520 & 21 & 4000 & 78,620 & 82,620 & 22 & 5000 & 73,454 & 78,454 & 21 & 5000 & 69,940 & 74,940 \\
\hline 14 & & 4454 & 21 & 4000 & 79,740 & 83,740 & 18 & 4000 & 72,300 & 76,300 & 21 & 5000 & 72,640 & 77,640 & 18 & 5000 & 64,900 & 69,900 \\
\hline 15 & \multirow[b]{2}{*}{ Tonge } & 160 & 26 & 4000 & 87,750 & 91,750 & 23 & 4000 & 79,680 & 83,680 & 26 & 5000 & 75,280 & 80,280 & 23 & 5000 & 70,560 & 75,560 \\
\hline 16 & & 168 & 24 & 4000 & 82,920 & 86,920 & 22 & 4000 & 78,440 & 82,440 & 24 & 5000 & 73,502 & 78,502 & 22 & 5000 & 69,360 & 74,360 \\
\hline
\end{tabular}




\section{Analysis of Results and Discussion}

This section provides a detailed analysis of the results presented in Tables 4-6. Moreover, the results are discussed in terms of the contribution to the literature.

Analyzing the results presented in Table 4 shows that the SA algorithm has been successful in achieving the optimal solutions in most of the test problems except for a few problems. In some cases where the optimal solution could not be found, the gap was negligible, which could be justified by the complexity of the problem. According to this table, for SIC $=500$, the SA has been capable of finding the optimal $T C$ for all the problems considering the $P S P=4$. For $P S P=5$, the optimal solution was found for 13 out of 16 problems ( $81 \%$ of the problems). Similarly, for SIC $=1000$, considering $P S P=4$, the SA algorithm was capable of finding the optimum solution for all the problems. For PSP $=5$, the optimum solution was obtained in 9 out of 16 problems. For the other seven problems or which the optimal solution could not be found, the gaps are less than $2.7 \%$. According to these results, it can be concluded that the performance of the SA algorithm proposed, in terms of finding the (near) optimal solutions, is promising, particularly by taking into account its minimal computational time, which ranged between 1 and $5 \mathrm{~s}$ for small and large size problems, respectively.

By analyzing the results reported in Tables 5 and 6, it can be seen that both $S C$ and TC are reduced when the SLP was solved after balancing the workload at stations. These results can verify the argument that optimal workload balancing can positively affect the resolution of SLP. Moreover, the comparison of results obtained for SLP after balancing the workload and before balancing indicates that for SIC $=500$ the TC has improved by $5.2 \%$ and $5.5 \%$ for $P S P=4$ and $P S P=5$, respectively. For SIC $=1000, T C$ has been enhanced by $5.2 \%$ and $4.6 \%$ for PSP $=4$ and PSP $=5$, respectively. Additionally, comparison of SLP solutions after and before balancing the workload for both SIC $=500$ and SIC $=1000$, show that the improvement in TC has been mainly caused by an improvement in $S C$ rather than IC (i.e., equal IC while $S C$ has almost improved in the majority of the test problems solved). Additionally, for SIC $=500$, the comparison of SLP solutions after balancing the workload for $P S P=4$ and $P S P=5$, shows that the increase of the potential number of supermarkets has resulted in $81 \%$ ( 13 out of 16 problems) decrease in TC. The same effect can be seen for SIC $=1000$ where a similar comparison shows $56 \%$ (9 out of 16) reduction in TC. This improvement can be justified to be the direct result of more freedom in choosing the location for establishing supermarkets, which leads to lesser transportation.

To provide a more detailed synthesis of results obtained, a comparison measure, known as the Relative Percent Deviation (RPD), is defined and presented as Equation (11). Using this measure, the relative percent deviation of different cost terms, namely installation cost (IC), shipment cost (SC), and total costs (TC) for comparing two scenarios (i.e., Feasible ALBP + SLP with Optimal ALBP + SLP) can be calculated.

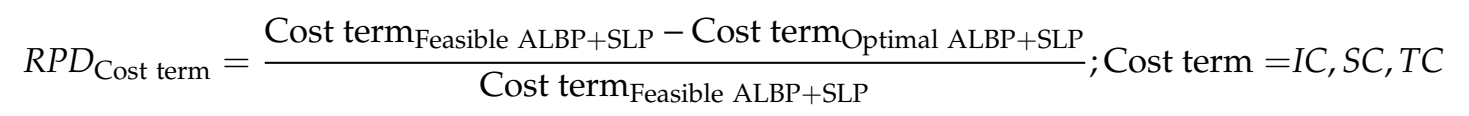

Figures 5 and 6 show the comparison of Feasible ALBP + SLP with Optimal ALBP + SLP in terms of $R P D_{I C}, R P D_{S C}$, and $R P D_{T C}$ for supermarket installation cost $(S I C)$ of 500 and 1000, respectively, as well as $P S P=4$ and $P S P=5$. In both figures, positive $R P D(\%)$ shows improvement, while negative $R P D(\%)$ shows a dis-improvement in the relating cost term.

According to Figure 5, for $P S P=4$ the amount of improvement in $R P D_{T C}$ is mainly caused by improvement in $R P D_{S C}$. For $P S P=5$, again $R P D_{S C}$ improvement has brought about the improvement in $R P D_{T C}$. It is worth noting that for $P S P=5$, despite negative $R P D_{I C}$ in problem 3 the positive $R P D_{S C}$ has led to positive $R P D_{T C}$ (i.e., $+6.7 \%$ ) in total.

Figure 6 also shows the same pattern that the improvement of $R P D_{S C}$ led to an improvement in $R P D_{T C}$ with only one exception for $P S P=4$, problem 2 , where the positive $R P D_{I C}$ dominated the negative $R P D_{S C}$ resulting in positive $R P D_{T C}$ (i.e., +4.6 ). 

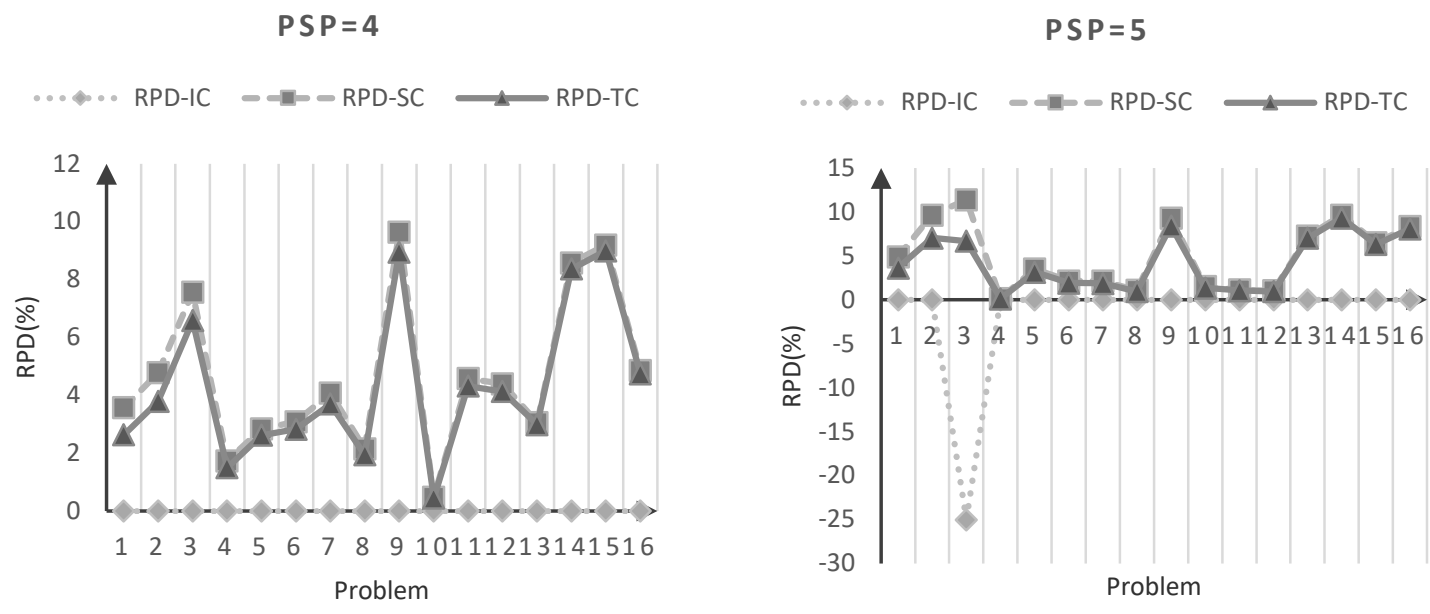

Figure 5. Comparison of Feasible ALBP + SLP and Optimal ALBP + SLP in terms of $R P D_{I C}, R P D_{S C}$ and $R P D_{T C}$ for $S I C=500$.
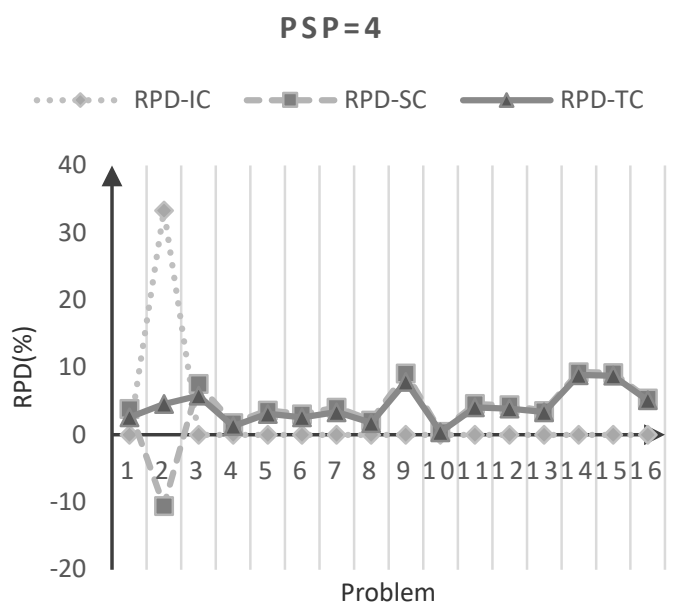

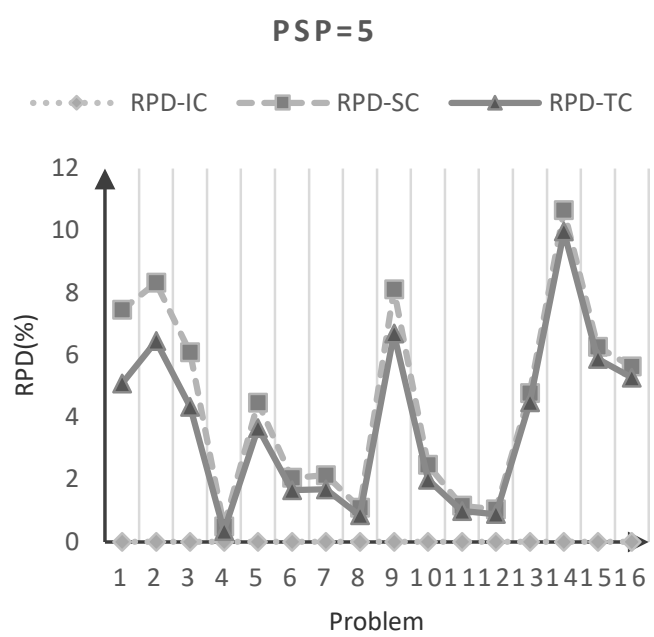

Figure 6. Comparison of Feasible ALBP + SLP and Optimal ALBP + SLP in terms of $R P D_{I C}, R P D_{S C}$ and $R P D_{T C}$ for $S I C=1000$.

\section{Conclusions}

To promote sustainability in the daily production processes, managers look for the best methods when dealing with the long-term decision problem of the assembly line design, specifically in terms of locating supermarkets. Additionally, the supermarket-concept has been recently adopted by many manufacturers to allow flexible and reliable Just-in-Time material delivery to assembly lines. Thus, this study aimed to optimize the configuration of assembly lines by considering the supermarket location problem known as SLP. For this purpose, the SA algorithm was developed to address SLP. The computational results on known test problems over the two possible supermarket places and two installation costs combinations verified that the proposed algorithm could optimize the configuration of the assembly lines from SLP considerations in terms of supermarket transportation and installation costs in a variety of test problems. Moreover, the effect of workload balancing on the solution of SLP was also investigated. The results revealed that the workload balancing reduces the total supermarket cost significantly and contributes to the overall environmental sustainability and economic sustainability of manufacturers by promoting production sustainability.

\section{Limitation and Future Directions}

The study might be considered limited in the sense that it benefited from the SA algorithm to address SLP. As future research directions, other meta-heuristic algorithms can be applied to solve the same 
problem and benchmarked against the SA algorithm developed in this study. Moreover, the present study and the algorithm developed could not, conceivably, incorporate the complete stochastic nature of production processes and activities within the manufacturing industry. Consistently, the stochastic nature of the production and material demand can also be taken into consideration in future studies. Additionally, the present study was developed based on the assumption of unique bin size and supermarket capacity, while future research can relax this particular assumption by considering bins with different sizes and supermarkets with different capacities. More importantly, and to address any limitations that the present study might have regarding the scope of production-related problems, investigating the effect of SLP on other operational decision problems such as routing, scheduling, and loading problems and their impacts on various dimensions of sustainability can also be an interesting research area for the future research.

Author Contributions: Conceptualization, M.F., A.N., M.G., and M.Y.; Formal analysis, M.F., and A.N.; Methodology, M.F. and A.N.; Project administration, A.N., M.G., and M.Y.; Supervision, M.F.; Writing-original draft, M.F., A.N. and M.G.; Writing—review and editing, M.F., M.G. and M.Y. All authors have read and agreed to the published version of the manuscript.

Funding: This research was funded by the KK-stiftelsen (Knowledge Foundation, Stockholm, Sweden) for the ProSpekt 2018 project OPTION.

Acknowledgments: The authors would like to thank the industrial partner for facilitating the site visits and providing insight into the problem through several meetings and discussions.

Conflicts of Interest: The authors declare no conflict of interest.

\section{References}

1. Cai, W.; Lai, K.-H.; Liu, C.; Wei, F.; Ma, M.; Jia, S.; Lv, L. Promoting sustainability of manufacturing industry through the lean energy-saving and emission-reduction strategy. Sci. Total Environ. 2019, 665, 23-32. [CrossRef] [PubMed]

2. Oluyisola, O.E.; Sgarbossa, F.; Strandhagen, J.O. Smart Production Planning and Control: Concept, Use-Cases and Sustainability Implications. Sustainability 2020, 12, 3791. [CrossRef]

3. Niaki, M.K.; Torabi, S.A.; Nonino, F. Why manufacturers adopt additive manufacturing technologies: The role of sustainability. J. Clean. Prod. 2019, 222, 381-392. [CrossRef]

4. Realyvásquez-Vargas, A.; Arredondo-Soto, K.C.; Blanco-Fernandez, J.; Sandoval-Quintanilla, J.D.; Jiménez-Macías, E.; García-Alcaraz, J.L. Work Standardization and Anthropometric Workstation Design as an Integrated Approach to Sustainable Workplaces in the Manufacturing Industry. Sustainability 2020, 12, 3728. [CrossRef]

5. Braccini, A.M.; Margherita, E.G. Exploring organizational sustainability of industry 4.0 under the triple bottom line: The case of a manufacturing company. Sustainability 2019, 11, 36. [CrossRef]

6. Zhang, Z.; Tang, Q.; Li, Z.; Zhang, L. Modelling and optimisation of energy-efficient U-shaped robotic assembly line balancing problems. Int. J. Prod. Res. 2019, 57, 5520-5537. [CrossRef]

7. Fathi, M.; Fontes, D.B.M.M.; Urenda Moris, M.; Ghobakhloo, M. Assembly line balancing problem: A comparative evaluation of heuristics and a computational assessment of objectives. J. Model. Manag. 2018, 13, 455-474. [CrossRef]

8. Ghafoorpoor Yazdi, P.; Azizi, A.; Hashemipour, M. A hybrid methodology for validation of optimization solutions effects on manufacturing sustainability with time study and simulation approach for SMEs. Sustainability 2019, 11, 1454. [CrossRef]

9. Fathi, M.; Nourmohammadi, A.; Ng, A.H.C.; Syberfeldt, A.; Eskandari, H. An Improved Genetic Algorithm with Variable Neighborhood Search to Solve the Assembly Line Balancing Problem. Eng. Comput. 2020, 37, 501-521. [CrossRef]

10. Fathi, M.; Nourmohammadi, A.; Ng, A.H.C.; Syberfeldt, A. An optimization model for balancing assembly lines with stochastic task times and zoning constraints. IEEE Access 2019, 7, 32537-32550. [CrossRef]

11. Ghobakhloo, M.; Fathi, M. Corporate survival in Industry 4.0 era: The enabling role of lean-digitized manufacturing. J. Manuf. Technol. Manag. 2019, 31, 1-30. [CrossRef] 
12. Battini, D.; Boysen, N.; Emde, S. Just-in-Time supermarkets for part supply in the automobile industry. J. Manag. Control 2013, 24, 209-217. [CrossRef]

13. Emde, S.; Gendreau, M. Scheduling in-house transport vehicles to feed parts to automotive assembly lines. Eur. J. Oper. Res. 2017, 260, 255-267. [CrossRef]

14. Kundu, K.; Rossini, M.; Portioli-Staudacher, A. A study of a kanban based assembly line feeding system through integration of simulation and particle swarm optimization. Int. J. Ind. Eng. Comput. 2019, 10, 421-442. [CrossRef]

15. Kong, L.; Li, H.; Luo, H.; Ding, L.; Zhang, X. Sustainable performance of just-in-time (JIT) management in time-dependent batch delivery scheduling of precast construction. J. Clean. Prod. 2018, 193, 684-701. [CrossRef]

16. Emde, S.; Boysen, N. Optimally locating in-house logistics areas to facilitate JIT-supply of mixed-model assembly lines. Int. J. Prod. Econ. 2012, 135, 393-402. [CrossRef]

17. Nourmohammadi, A.; Eskandari, H.; Fathi, M.; Ng, A.H. Integrated locating in-house logistics areas and transport vehicles selection problem in assembly lines. Int. J. Prod. Res. 2020,1-19. [CrossRef]

18. Alnahhal, M.; Noche, B. A genetic algorithm for supermarket location problem. Assem. Autom. 2015, 35, 122-127. [CrossRef]

19. Zacharia, P.T.; Nearchou, A.C. A population-based algorithm for the bi-objective assembly line worker assignment and balancing problem. Eng. Appl. Artif. Intell. 2016, 49, 1-9. [CrossRef]

20. Chen, J.; Wang, J.; Baležentis, T.; Zagurskaitè, F.; Streimikiene, D.; Makutenienè, D. Multicriteria Approach towards the Sustainable Selection of a Teahouse Location with Sensitivity Analysis. Sustainability 2018, 10, 2926. [CrossRef]

21. Zeng, S.; Nestorenko, O.; Nestorenko, T.; Morkūnas, M.; Volkov, A.; Baležentis, T.; Zhang, C. EOQ for perishable goods: Modification of Wilson's model for food retailers. Technol. Econ. Dev. Econ. 2019, 25, 1413-1432. [CrossRef]

22. Li, Z.; Kucukkoc, I.; Nilakantan, J.M. Comprehensive review and evaluation of heuristics and Meta-heuristics for two-sided assembly line balancing problem. Comput. Oper. Res. 2017, 84, 146-161. [CrossRef]

23. Eghtesadifard, M.; Khalifeh, M.; Khorram, M. A systematic review of research themes and hot topics in assembly line balancing through the web of science within 1990-2017. Comput. Ind. Eng. 2020, 139, 106-182. [CrossRef]

24. Battini, D.; Faccio, M.; Persona, A.; Sgarbossa, F. Supermarket warehouses: Stocking policies optimization in an assembly-to-order environment. Int. J. Adv. Manuf. Technol. 2010, 50, 775-788. [CrossRef]

25. Nourmohammadi, A.; Eskandari, H. Assembly line design considering line balancing and part feeding. Assem. Autom. 2017, 37, 135-143. [CrossRef]

26. Nourmohammadi, A.; Eskandari, H.; Fathi, M.; Aghdasi, M. A mathematical model for supermarket location problem with stochastic station demands. In Proceedings of the CIRP: 51st CIRP Conference on Manufacturing Systems, Stockholm, Sweden, 16-18 May 2018; Volume 72, pp. 444-449.

27. Nourmohammadi, A.; Eskandari, H.; Fathi, M. Design of stochastic assembly lines considering line balancing and part feeding with supermarkets. Eng. Optim. 2019, 51, 63-83. [CrossRef]

28. Zhou, B.-H.; Tan, F. A Self-Adaptive Estimation of Distribution Algorithm with Differential Evolution Strategy for Supermarket Location Problem. Neural Comput. Appl. 2019, 32, 5791-5804. [CrossRef]

29. Kirkpatrick, S.; Gelet, C.D.; Vecchi, M.P. Optimization by simulated annealing. Science 1983, 220, $621-630$. [CrossRef]

30. Fathi, M.; Álvarez, M.J.; Rodríguez, V. A new heuristic-based bi-objective simulated annealing method for U-shaped assembly line balancing. Eur. J. Ind. Eng. 2016, 10, 145-169. [CrossRef]

31. Fathi, M.; Alvarez, M.J.; Mehraban, F.H.; Rodríguez, V. A Multiobjective Optimization Algorithm to Solve the Part Feeding Problem in Mixed-model Assembly Lines. Math. Probl. Eng. 2014, 1, 1-12. [CrossRef]

32. Xia, X.; Liu, W.; Zhang, Z.; Wang, L.; Cao, J.; Liu, X. A Balancing Method of Mixed-model Disassembly Line in Random Working Environment. Sustainability 2019, 11, 2304. [CrossRef]

(C) 2020 by the authors. Licensee MDPI, Basel, Switzerland. This article is an open access article distributed under the terms and conditions of the Creative Commons Attribution (CC BY) license (http://creativecommons.org/licenses/by/4.0/). 